\title{
Journal of NeuroEngineering and Rehabilitation reviewer acknowledgement 2014
}

David J Reinkensmeyer $r^{1,2,3,4}$

\section{Contributing reviewers}

The editors of Journal of NeuroEngineering and Rehabilitation would like to thank all of our reviewers who have contributed to the journal in volume 11 (2014).

\author{
Daijiro Abe \\ Japan \\ U. Rajendra Acharya \\ Singapore
}

Diane E. Adamo

United States of America

Sergei Adamovich

United States of America

\section{Allan Adkin \\ Canada}

Valentina Agostini

Italy

Zaghloul Ahmed

United States of America

Mark Albert

United States of America

Joan F. Alonso

Spain

Farshid Amirabdollahian

United Kingdom

Philippe Archambault

Canada

\section{J. Hans Arendzen \\ Netherlands}

John Arkwright

Australia

Panagiotis Artemiadis

United States of America

Alexander Aruin

United States of America

Oskar Aszmann

Austria

Musa Audu

United States of America

Louis Awad

United States of America

Ali Bahramisharif

Netherlands

\section{Alberto Baldini}

Italy

Subhasis Banerji

Singapore

Robert John Barry

Australia
Dorothy Barthélemy
Canada

Gabor Barton

United Kingdom

Rezaul Begg

Australia

Nikunj Bhagat

United States of America

Tanvi Bhatt

United States of America

Daniele Bibbo

Italy

Sandra Billinger

United States of America

Martin Bilodeau

Canada

Stuart Binder-Macleod United States of America

Andreanne Blanchette

Canada

Amy Blank

United States of America

Correspondence: jner@biomedcentral.com

${ }^{1}$ Department of Mechanical and Aerospace Engineering, University of California, Irvine, USA

${ }^{2}$ Department of Anatomy and Neurobiology, University of California, Irvine, USA

${ }^{3}$ Department of Biomedical Engineering, University of California, Irvine, USA

${ }^{4}$ Department of Physical Medicine and Rehabilitation, University of California, Irvine, USA 
Florian Bodranghien

Belgium

Richard Bohannon

United States of America

Matthieu Boisgontier

Belgium

Frank Borg

Finland

Jörgen Borg

Sweden

Dennis Bourbeau

United States of America

Mark Bowden

United States of America

John-Stuart Brittain

United Kingdom

Cynthia Brown

United States of America

Dustin Bruening

United States of America

Michelle Burack

United States of America

Etienne Burdet

United Kingdom

Monica Cameirao

Portugal

Filippo Camerota

Italy

Leonardo Cappello

Italy

Stephanie Carey

United States of America

Tom Carlson

United Kingdom

Stephen Carp

United States of America

Helder Carvalho

Portugal

Margherita Castronovo

Germany

Brian Caulfield

Ireland

Massimo Cenciarini

Germany
Rosa H. M. Chan

Hong Kong

Shuo-Hsiu Chang

United States of America

Yao-Jen Chang

Taiwan

Xiang Chen

China

Félix Chénier

Canada

Carmelo Chisari

Italy

Veronica Cimolin

Italy

Pietro Cipresso

Italy

Edward (Ted) Clancy

United States of America

Ross Clark

Australia

Sue Cobb

United Kingdom

Steve Collins

United States of America

Silvia Conforto

Italy

Elaine Corbett

Australia

Patrick Costigan

Canada

Elaine Coulter

United Kingdom

Patrick Crago

United States of America

Frederic Crevecoeur

Canada

Dustin Crouch

United States of America

Xingran Cui

United States of America

Armin Curt

Switzerland

Marco D'Alonzo

Italy
Carmen D'Anna

Italy

Paulo de Freitas

Brazil

Ray de Leon

United States of America

Ana de los Reyes Guzmán Spain

Helios de Rosario

Spain

Jesse Dean

United States of America

Philippe Dedieu

France

Ugo Della Croce

Italy

Thibault Deschamps

France

Nandini Deshpande

Canada

Judith Deutsch

United States of America

Michael Devlin

Canada

Nuno Dias

Portugal

Dan Ding

United States of America

An Do

United States of America

Christian Dohle

Germany

Takehiko Doi

Japan

Strahinja Dosen

Germany

Jennifer Dunn

New Zealand

Lucy Dunne

United States of America

Assaf Dvorkin

United States of America

Gerold Ebenbichler

Austria 
Gail Eskes

Canada

Debbie Espy

United States of America

Alberto Esquenazi

United States of America

Alfonso Fasano

Canada

Philippe Faure

France

Xin Feng

United States of America

Jing Feng

United States of America

Simona Ferrante

Italy

Giancarlo Ferrigno

Italy

Daniel Ferris

United States of America

Peter Feys

Belgium

Fanny Ficuciello

Italy

Hillel Finestone

Canada

James Finley

United States of America

Lee Fisher

United States of America

Kevin Fite

United States of America

Bertine Fleerkotte

Netherlands

Robert Flint

United States of America

Gerard Fluet

United States of America

Stephen Foldes

United States of America

Kenneth Fong

Hong Kong

Emily Fox

United States of America
Jason Friedman

Israel

Gail Frost

Canada

David Gabriel

Canada

Alejandro Galan-Mercant

Spain

Sujay Galen

United States of America

Philippe Gallien

France

Brook Galna

United Kingdom

Fan Gao

United States of America

Nicolas Garcia Aracil

Spain

Laura Gastaldi

Italy

Lynne Gauthier

United States of America

Xinling Geng

China

Alireza Gharabaghi

Germany

Giuseppina Gini

Italy

Leonardo Gizzi

Germany

Manuel González-Sánchez

Spain

Keith Gordon

United States of America

Alena Grabowski

United States of America

Robert Gregg

United States of America

Philip Grewe

Germany

Michael Grey

United Kingdom

Giuliana Grimaldi

Belgium
Eleni Grimpampi

Italy

Emmanuel Guigon

France

Etienne Guillaud

France

Lan-Yuen Guo

Taiwan

Michael Hahn

United States of America

Courtney Hall

United States of America

Gyu Cheol Han

South Korea

Noor Hanif

United Kingdom

Andrew Hansen

United States of America

Orla Hardiman

Ireland

Levi Hargrove

United States of America

Chris Hass

United States of America

Christopher Hasson

United States of America

Anna Hatton

Australia

Malcolm Hawken

United Kingdom

Daniel Heitzmann

Germany

Trent Herda

United States of America

Stefan Hesse

Germany

Shivayogi Hiremath

United States of America

Ales Holobar

Slovenia

Tiago Hori

Canada

Elizabeth Hsiao-Wecksler

United States of America 
Ru-Lan Hsieh

Taiwan

Jwu-Sheng Hu

Taiwan

Yong $\mathbf{H u}$

Hong Kong

Hsiu-Chen Huang

Taiwan

He (Helen) Huang

United States of America

Christopher Hurt

United States of America

Ing-Shiou Hwang

Taiwan

Trienke IJmker

Netherlands

Marco Iosa

Italy

Md. Anamul Islam

Malaysia

Mark Ison

United States of America

Nathanael Jarrassé

France

Steve Jax

United States of America

Arun Jayaraman

United States of America

Jean-Yves Jenny

Fiji

Winnie Jensen

Denmark

Ning Jiang

Germany

Reva Johnson

United States of America

Alon Kalron

Israel

Derek Kamper

United States of America

Guiyeom Kang

Ireland

Shigehisa Kawakami

Japan
Stephen Kelly

United Kingdom

Trisha Kesar

United States of America

Rami Khushaba

Australia

Kevin Kilgore

United States of America

Gyoung-Mo Kim

South Korea

Sohee Kim

South Korea

Kristof Kipp

United States of America

Rachel Kizony

Israel

Verena Klamroth-Marganska

Switzerland

Bert Kleine

Netherlands

Rudolf Klemetti

Finland

Marco Knaflitz

Italy

Maria Knikou

United States of America

Toshiki Kobayashi

United States of America

Silvia Erika Kober

Austria

Dylan Kobsar

Canada

Rodger Kram

United States of America

Sharon Kramer

Australia

Hermano Igo Krebs

United States of America

Carmen Krewer

Germany

Hollis Krug

United States of America

Todd Kuiken

United States of America
Dinesh Kumar

Australia

Jason Kutch

United States of America

Laurel Kuxhaus

United States of America

Ruben Kuzniecky

United States of America

Peter Kyberd

Canada

Joris Lambrecht

United States of America

Claudine Lamoth

Netherlands

Beom Chan Lee

United States of America

Hyunglae Lee

United States of America

Sang Wook Lee

United States of America

Yun-Ju Lee

United States of America

Yafi Levanon

Israel

Mindy Levin

Canada

Beth Lewandowski

United States of America

Sandra Lewis

United Kingdom

Jan Lexell

Sweden

Guanglin Li

China

Karen Li

Canada

Ke Li

China

Li Li

United States of America

Meiling Li

China

Qingguo Li

Canada 
Joachim Liepert

Germany

Fang Lin

United States of America

Jonathan Lin

Canada

Suh-Jen Lin

United States of America

Pavel Lindberg

France

Simon Little

United Kingdom

Beth Lopour

United States of America

Stephen Lord

Australia

Richard Lovering

United States of America

Madeleine Lowery

Ireland

Marilyn MacKay-Lyons

Canada

Andreas Maier

Germany

Margaret Mak

Hong Kong

Philippe Malcolm

Belgium

Martina Mancini

United States of America

Brad Manor

United States of America

Mario Manto

Belgium

Marin Manuel

France

Robin Marcus

United States of America

Susan Marion

United States of America

\section{Alain Martin}

France

Joel Martin

United States of America
Roshan Joy Martis

Singapore

Sarah Mason

United Kingdom

Giulia Matrone

Italy

Alessandro Mauro

Italy

Claudia Mazzà

Italy

Suzanne McDonough

United Kingdom

Bradford McFadyen

Canada

Paul McGeoch

United States of America

Kevin McGill

United States of America

Linda McLean

Canada

Ranjana Mehta

United States of America

Sabato Mellone

Italy

William Memberg

United States of America

Rochelle Mendonca

United States of America

Thomas Mergner

Germany

Anat Mirelman

Israel

Sambit Mohapatra

United States of America

Filippo Molinari

Italy

Marco Molinari

Italy

Luis Montesano

Spain

J. Leon Morales-Quezada

United States of America

Juan C. Moreno

Spain
Steven Morrison

United States of America

Winfred Mugge

Netherlands

Gernot Müller-Putz

Austria

Sandro Mussa-Ivaldi

United States of America

Kristin Musselman

Canada

Pratik Mutha

India

Jiro Nakano

Japan

Dominic Nathan

United States of America

Tobias Nef

Switzerland

Francesco Negro

Germany

Elizabeth Nightingale

Australia

Domen Novak

Switzerland

Toru Ogata

Japan

Shinya Ogaya

Japan

Lauro Ojeda

United States of America

Taher Omari

Australia

Kenneth Ottenbacher

United States of America

Kerstin Palombaro

United States of America

Enrica Papi

United Kingdom

Matthew Parkes

United Kingdom

Ilaria Pasciuto

Italy

Ben Patritti

Australia 
Carolynn Patten

United States of America

Kara Patterson

Canada

Lorna Paul

United Kingdom

Alessandra Pedrocchi

Italy

Joel Perry

Spain

Angel Peterchev

United States of America

Mark Peterson

United States of America

Daniel Peterson

United States of America

Gert Pfurtscheller

Austria

Elena Pirogova

Australia

Mark Pitkin

United States of America

Dejan Popovic

Serbia

Mira Popovic

Serbia

Sigal Portnoy

Israel

Martin Posch

Austria

Gerdienke B. Prange

Netherlands

Rachel Proffitt

United States of America

Alessio Pulizzotto

Italy

Kai Qian

United States of America

Robrecht Raedt

Belgium

Danny Rafferty

United Kingdom

Fabian Rast

Switzerland
Rafael Raya

Spain

Jeffrey Reinbolt

United States of America

Olivier Remy-Neris

France

Daniel Renjewski

Switzerland

Christopher Rhea

United States of America

Sarah Ridge

United States of America

Laura Rocchi

Italy

Eduardo Rocon

Spain

Eric Rombokas

United States of America

Noah Rosenblatt

United States of America

Diana Ruiz Bueno

Spain

Ruediger Rupp

Germany

David Russ

United States of America

Manning Sabatier

United States of America

Hooshang Saberi

Iran

Brian Sandroff

United States of America

Amirehsan Sarabadani Tafreshi

Switzerland

Makoto Sasaki

Japan

Katherine Saul

United States of America

Zimi Sawacha

Italy

Lumy Sawaki

United States of America

Andrew Sawers

United States of America
Gregory Sawicki

United States of America

Edward Sazonov

United States of America

Sydney Schaefer

United States of America

Philip Schatz

United States of America

Erik Scheme

Canada

Sheila Schindler-Ivens

United States of America

Maurizio Schmid

Italy

Peter Schneider

Germany

Alfred Schouten

Netherlands

Stylianos Scordilis

United States of America

Stephen Scott

Canada

Thomas Seel

Germany

Ava Segal

United States of America

Ervin Sejdic

United States of America

Masaki Sekine

Japan

Jon Sensinger

Canada

Na Jin Seo

United States of America

Giacomo Severini

United States of America

Ludy Shih

United States of America

Pete Shull

China

Kathryn Sibley

Canada

Ann Simon

United States of America 


\begin{tabular}{|c|c|c|}
\hline Mikhail Simonov & Alessandro Tognetti & Jinsung Wang \\
\hline Italy & Italy & United States of America \\
\hline Tarkeshwar Singh & Craig Tokuno & Yijun Wang \\
\hline United States of America & Canada & United States of America \\
\hline Sue Ann Sisto & Diego Torricelli & William Warren \\
\hline United States of America & Spain & United States of America \\
\hline Manoj Sivan & Yannick Tousignant-Laflamme & Kathleen Watson \\
\hline United Kingdom & Canada & United States of America \\
\hline Lauren Smith & Diana Trojaniello & Seng Kwee Wee \\
\hline United States of America & Italy & Singapore \\
\hline Marco Soares dos Santos & Randy Trumbower & Joseph Weir \\
\hline Portugal & United States of America & United States of America \\
\hline Jacob Sosnoff & Inna Tsirlin & Aner Weiss \\
\hline United States of America & Canada & Israel \\
\hline Patrick Sparto & Carole Tucker & Jill Whitall \\
\hline United States of America & United States of America & United States of America \\
\hline Shmuel Springer & Andrea Turolla & Maura Whittaker \\
\hline Israel & Italy & Canada \\
\hline Daniel Stashuk & Ailie Turton & Jason Wingert \\
\hline Canada & United Kingdom & United States of America \\
\hline Despina Stavrinos & Edwin van Asseldonk & Hartwig Woldag \\
\hline United States of America & Netherlands & Germany \\
\hline Joel Stein & Herman van der Kooij & Shun-Chi Wu \\
\hline United States of America & Netherlands & Taiwan \\
\hline Jill Stewart & Bram van Dun & Ming Wu \\
\hline United States of America & Australia & United States of America \\
\hline Ian Stokes & Richard van Emmerik & Jianhui Wu \\
\hline United States of America & United States of America & China \\
\hline Oliver Stoller & Natalie Vanicek & Max Wuehr \\
\hline Switzerland & United Kingdom & Germany \\
\hline Jake Streepey & Giovanni Vecchiato & Sophie Marie Wurth \\
\hline United States of America & Italy & Switzerland \\
\hline Fong-Chin Su & Beatrix Vereijken & Hongbo Xie \\
\hline Taiwan & Norway & Australia \\
\hline Sandeep Subramanian & Paul F.M.J. Verschure & Feng Xu \\
\hline Canada & Spain & China \\
\hline Tomohiko Takei & Bruce Volpe & Zhongliang Yang \\
\hline Japan & United States of America & China \\
\hline Andrew Tan & Tim Wagner & Jun Yao \\
\hline United States of America & United States of America & United States of America \\
\hline Nitish Thakor & Erik Walbeehm & Aaron Young \\
\hline United States of America & Netherlands & United States of America \\
\hline Michael Thaut & Conrad Wall & Nuray Yozbatiran \\
\hline United States of America & United States of America & United States of America \\
\hline
\end{tabular}


Bing Yu

United States of America

Karl Zabjek

Canada

Vladimir Zatsiorsky

United States of America

Karl Zelik

United States of America
Erika Zemková

Slovakia

Joseph Zeni

United States of America

Mingming Zhang

New Zealand

Qin Zhang

China
Xu Zhang

China

Yingchun Zhang

United States of America

Jun-Tian Zhang

Canada 